\title{
Deployment and Future Prospects of High Performance Diagnostics featuring Serial I/O (SIO) Data Acquisition (DAQ) at ASDEX Upgrade
}

\author{
K. Behler ${ }^{1}$, H. Blank ${ }^{1}$, H. Eixenberger ${ }^{1}$, M. Fitzek ${ }^{2}$, A. Lohs ${ }^{1}$, K. Lüddecke², R. Merkel ${ }^{1}$, \\ ASDEX Upgrade Team
}

${ }^{1}$ Max-Planck-Institut für Plasmaphysik, Boltzmannstr. 2, D-85748 Garching bei München

${ }^{2}$ Unlimited Computer Systems GmbH, Seeshaupterstr. 15, D-82393 Iffeldorf

\begin{abstract}
The SIO DAQ concept used at the ASDEX Upgrade fusion experiment features data acquisition from a modular front-end (a modular crate-and-interface-cards concept for analog and digital input and output) over standardized serial lines and via a serial input/output computer interface card (the SIO card) in real-time directly into the main memory of a host computer.
\end{abstract}

Deployment of a series of diagnostics using SIO led to various solutions and configurations for the different requirements. Experience has been gained and lessons learned applying the SIO concept at its technical limits. Requirements for a further development of the SIO concept have been identified, and a performance improvement by a factor of 4-8 beyond its current limits seems achievable. An effort has been started to develop a SIO version 2 (SIO II) featuring upgraded serial links and a more powerful FPGA for merging and forwarding data streams to host computer memory. (Compatibility with the existing SIO (SIO I) front-end system has to be maintained.)

This paper presents results achieved and experiences gained in the deployment of SIO I, the status of SIO II development (currently in the prototype phase), and projected enhancements and updates to existing implementations.

\section{Introduction}

The SIO DAQ concept was developed to provide a simple and versatile method for connecting external measuring systems to computers via standard serial links. Its main features are four bidirectional HOTLink data ports and a central timing clock port (all fiber optics technology), a central FPGA containing the data and time merge logic, and a fast PCI or PCIexpress computer interface. The FPGA as the "heart" of the SIO card merges four incoming streams of samples and the internal stream of time stamps to time-stamped data frames. These are transferred frame by frame with lowest possible latency via the PCI interface into the shared memory of the host computer providing immediate access to the data by application programs. This concept together with the in house developed analogue to digital conversion (ADC) front-end configuration was described earlier [1][2]. In 2009 this concept was chosen to become the new standard for ASDEX Upgrade diagnostics. (Similar concepts have been developed independently by others [3]. Differing DAQ I/O concepts like Ethernet based ADC devices or ADC carrying interface cards (PCI, PCIe, cPCI, PXI, ATCA or $\mu$ TCA) shall not be discussed in this paper, since the justification of the decision to go the SIO direction with the described modular external front-end configuration for ASDEX Upgrade has been presented earlier [2].)

While a few bulk data diagnostics (up to 128 channels, 10-500 kHz sampling frequency) have been set-up using the SIO concept, it was recognized that other more challenging diagnostics could also benefit from the SIO technology but would need to exploit its data transport capabilities to the limit. The Electron Cyclotron Emission (ECE, internal name RMC) and the Doppler-Reflectometry (internal name PRA) diagnostic have been deployed pushing current SIO I hardware to its technical limits. For the PRA diagnostic, an additional FPGA software modification was necessary. 
In these highly tuned configurations, reliable operation is difficult to achieve. The complex multithreaded RT (real-time) software and system configuration is highly sensitive to small discrepancies from the optimal set-up. Enhancing the head room by designing a significantly more powerful SIO device seems desirable.

Considering the development of today's chip technology, enhancements are achievable by porting and refurbishing existing functionality to a new chip set, allowing for a dramatic increase in capabilities, features and performance. The PCI interface and the serial link handling, which were formerly located in separate chips, become integrated software blocks in the FPGA. The concomitant simplification of the board layout together with performance upgrades justify the higher price of an advanced FPGA. As the new "heart" of SIO II, we have selected a "mid-range" Altera Arria II GX [4]. This chip provides the desired data transfer capabilities for a faster and more flexible ${ }^{1}$ SIO engine and additionally incorporates the PCIe interface to the host and the serial connectivity to the front-ends in the form of IP (Intellectual Property) software blocks [5][6]. In this hardware configuration SIO II is projected to provide four bidirectional 2.5 Gbps serial optical links for input and a final data rate of up to 10 Gbps into the host.

\section{Recently Deployed SIO I Diagnostics}

SIO I as a new standard DAQ solution for diagnostics at ASDEX Upgrade (AUG) boosted the refurbishment, renewal and realization of old and new diagnostic projects. Table 1 gives a list of recently deployed AUG diagnostics realized with SIO I. In all cases DAQ is done by direct transfer

\begin{tabular}{|c|c|c|c|c|c|c|c|c|}
\hline Diagnostic & CPU cores & bus & \# SIO/links & pipelines $x$ slots & DAQ modules & sample rate & data rate & data size \\
\hline r-t $n_{e}$ Interferom. (DCN) & 1x sparcV9 & $\mathrm{PCl}$ & $1 / 1$ & $1 \times 12$ & $5 \times F r g C n t+3 \times 2$ chADCs & $50 \mathrm{kHz}$ & $1.6 \mathrm{MB} / \mathrm{s}$ & $17 \mathrm{M}$ \\
\hline r-t current profile (MSE) & $2 \times 4$ amd64 & $\mathrm{PCle}$ & $1 / 1$ & $1 \times 16$ & $13 \times 2 \mathrm{chADCs}$ & $250 \mathrm{kHz}$ & $15 \mathrm{MB} / \mathrm{s}$ & $180 \mathrm{~N}$ \\
\hline ECE (RMC) & $2 \times 4$ amd64 & $\mathrm{PCle}$ & $2 / 4+4$ & $2 \times 4 \times 2$ & $8 \times 2 \times 4$ chADC (wls) & $1 \mathrm{MHz}$ & $2 \times 72 \mathrm{MB} / \mathrm{s}$ & \\
\hline XUV emission (XVR) & 2x sparcV9 & PCle & $2 / 4+4$ & $2 \times 4 \times 16$ & 128x XUV-ADCs & $500 \mathrm{kHz}$ & $2 \times 68 \mathrm{MB} / \mathrm{s}$ & \\
\hline XUV emission (XVS) & 1x sparc $\mathrm{g}$ & $\mathrm{PCle}$ & $2 / 4+3$ & $(4+3) \times 16$ & 112x XUV-ADCs & $500 \mathrm{kHz}$ & $68+52 \mathrm{MB} / \mathrm{s}$ & $960 \mathrm{~N}$ \\
\hline VUV Spectr. (CCD) & 1x sparc $V 9$ & $\mathrm{PCl}$ & $4 / 1$ & custom CCD FE & 4x CCD-HL Adapter & $1 \mathrm{kHz}$ & $6 \mathrm{MB} / \mathrm{s}$ & 24 \\
\hline Fast Ion Loss & 1x sparc $V 9$ & $\mathrm{PCl}$ & $1 / 3$ & $3 \times 4$ & $9 \times 2$ chADCs & $1 \mathrm{MHz}$ & $44 \mathrm{MB} / \mathrm{s}$ & 352 \\
\hline Lithium Beam & $2 \times 4$ amd64 & $\mathrm{PCle}$ & $1 / 3$ & $3 \times 16$ & $48 \times 2$ chADCs & $200 \mathrm{kHz}$ & $40 \mathrm{MB} / \mathrm{s}$ & 27 \\
\hline Reflectometry fluct. & $2 \times 4$ amd64 & $\mathrm{PCle}$ & $1 / 1$ & $1 \times 4$ & $2 \times 2$ chADCs & $1 \mathrm{MHz}$ & $16 \mathrm{MB} / \mathrm{s}$ & 128 \\
\hline Doppler Reflectometry & $2 \times 4$ amd64 & PCle & $2 / 2$ & custom FE & 2 bands ( $2 \times 2$ ADCs) & $20 \mathrm{MHz}$ & $2 \times 80 \mathrm{MB} / \mathrm{s}$ & 1.2 \\
\hline
\end{tabular}

of data into computer memory using DMA (direct memory access). The DMA transfers are supervised by a real-time process responsible for preparing, starting and restarting a chain of DMA operations as necessary. The "green" table rows mark diagnostics running additional RT processes sharing the DAQ memory read-only. These diagnostics provide capabilities for RT analysis and RT communication with plasma control computers (Control). These RT analysis results serve as input or feedback for advanced plasma scenarios like density profile control [7] or MHD mode stabilization [8][9]. While the other listed diagnostics perform the actual data transfer to memory in real-time, the final archiving as AUG shot files is done - as usual - off-line after the plasma discharge.

As a further enhancement of the ECE diagnostic, it is projected to upgrade the sampling rate from 1 to $2 \mathrm{MHz}$ and possibly the number of channels from 64 to 68 or 72 . However, it is obvious that this upgrade is not possible with the current performance of the SIO I interface.

1 While the SIO I engine was restricted to a simple parametrized operation, the advanced SIO II engine allows fully programmable merge and transfer operation. 


\section{SIO I Performance}

A number of the above mentioned diagnostics are testing the performance of SIO I to the limits. Electron Cyclotron Emission (ECE) as well as XUV emission and Doppler Reflectometry have data rates very close to their nominal maximum computer bus speeds. In fact, ECE was initially designed as a split diagnostic on two hosts (internal names RM-A and -B - from Radio Meter), but by employing a more powerful computer, both SIO devices could be deployed in a single host named RMC. The XUV emission diagnostic (internal diagnostic names XVR and XVS) is still a distributed system with multiple SIO cards on multiple computer nodes. The Doppler Reflectometry features two SIO cards (one for each microwave band) with a modified SIO engine allowing a 20 $\mathrm{MHz}$ sampling rate. (Instead of one time stamp per clock tick, only one time stamp per measuring interval is generated.)

While the parallel set-up of "DAQ only" diagnostics with multiple host nodes is a simple way of managing high demands (the two XUV diagnostics just store data in two different shot-files), it would make things complex in cases where all channels of a diagnostic have to be processed in real-time to produce a result of relevance for Control. One would like to avoid the parallelism of distributed DAQ with subsequent distributed RT analysis applications. (The ECE hosts RMA \& RMB were combined into one host RMC for this reason.) RMC (see Table 1, line 3) using two SIO I devices being managed by two RT threads acquires all 64 radiometer channels into two shared memories on the same host. On the same host, an RT analysis task is keeping in step with the DMA reading from both shared segments. All 64 ECE channels can be examined for MHD activity signatures without having to resort to a distributed software solution.

On a single host computer, the RMC diagnostic system is able to acquire an incoming data stream at $144 \mathrm{MB} / \mathrm{s}$, analyze the data stream in 5 or $10 \mathrm{~ms}$ time slices, and send the results to the ASDEX Upgrade discharge control system (DCS) via the diagnostics to control communication protocol. [10]

The data rates achieved with SIO I are very close to the technical limits of the used communication channels. With dual-device set-ups (two SIO I in different I/O slots of the same computer) even higher data rates of $144 \mathrm{MB} / \mathrm{s}$ (RMC diagnostic) or $160 \mathrm{MB} / \mathrm{s}$ (Doppler Ref.) can be achieved. Both diagnostics are used regularly with these data rates in experiment operation.

\section{"Frame-drops" with SIO I at High Data Rates}

SIO does not use large internal buffers (see next section). The philosophy is to continously send all data by DMA immediately and smoothly (as a continous stream without pauses) into the main memory of the host computer. Under cutting edge conditions, which apply to some of our diagnostics, it is not easy to maintain the DMA streaming as smooth as required. Any interruption of the DMA caused by various reasons - reloading a virtual address table, activity of a high priority task or hardware device, delayed interrupt request (IRQ) handling because of other system activity will lead to a short stoppage of the data flow. These stoppages, if they reduce the DMA duty cycle considerably, may degrade the overall transfer speed. If they become even longer, they may cause internal SIO buffers to overflow and ultimately, the loss of SIO time frames. In the SIO context this is called a "frame-drop" or a series of frame-drops and has raised some discussions about the reliability and usefulness of the SIO concept. Frame-drops, how they occur, and what measures to take against them shall be discussed briefly.

The term "frame" has already been described in [1]. To make it more clear we would like to look deeper into the FPGA software blocks once again. Frames are assembled by the FPGA from the five primary SIO inputs: four from its serial links and a fifth internal stream of time-stamps from its synchronized clock. This is done by the "time engine" and the "merge engine" working together in triggering the external front-end and managing the serial links. Together they form the "SIO 
engine". The most important responsibility of the SIO engine is to keep data and time together correspondingly. To ensure this, each cycle of the SIO engine generates exactly one frame from the right pieces in the time FIFO and the four serial link FIFOs in a deterministic way. The completed frames are placed one by one into the DMA output FIFO to be transferred to the host's memory by the "DMA engine", which concurrently transfers frames out of the FIFO into host memory as requested by the DAQ task. If frames enter the FIFO faster than they are transferred out, the FIFO fills up, reducing the space available for new input frames. If the available space is not sufficient to store the next full frame, nothing is delivered into the FIFO and that entire frame is discarded, resulting in a frame drop. Frames will be dropped until enough data has been transferred out of the FIFO, freeing enough space to hold at least one entire frame. This policy guarantees that no incomplete frames will be delivered to the host.

\section{How Frame-Drops are Affected by Data Rate and System Parameters}

The DMA FIFO is small ( $32 \mathrm{~KB})$ by design, to avoid long latency times between production of data and transfer to the host, fulfilling the design goal of short latency from source to memory. A necessary condition for this mechanism to work well is to minimize interruptions to the DMA traffic to the host. The FIFO size divided by the targeted data rate directly yields the maximum allowed interruption time. In other words, the higher the data rate, the shorter the transfer interruptions may be.

To avoid DMA interruptions and frame-drops one could issue a single long DMA request for the full acquisition length without interruptions. (This is in fact done for the XUV and Doppler Reflectometry diagnostics.) But due to not understood synchronization issues between CPU and memory in the computer architecture, this seems not to be possible when a RT process wants concurrently to keep in step with the DMA progress. To make the data in shared memory available to other processes in real time, it appears necessary to break up the DMA into many short time slices. To avoid frame drops, it is essential that the next DMA request is started immediately upon finishing the preceding one. A careful tuning of the system configuration and the data stream parameters is necessary to achieve this. The measures taken to minimize delays in Solaris interrupt request (IRQ) handling are described below.

\section{How Frame-Drops Affect DAQ}

The SIO behavior to drop only full frames guarantees the delivered data structure can always be interpreted. Using the embedded time-stamps, the amount of lost data and the full time axis are always identifiable. Typically the drop-out time at the described maximum used data rates is less than $1 \mathrm{~ms}$ in 6-8 seconds of total acquisition time. So reliability is still better than $99.98 \%$.

Often a characteristic frame-drop pattern over time can be observed when approaching performance limits. Specific to the RMC diagnostic, for example, are 1 or 2 frame-drop intervals of approximately $500 \mu \mathrm{s}$ length during the first few milliseconds (the very beginning) of a plasma discharge. Any frame-drops lead to a non equidistant time axis of the data channels. A sophisticated time base handling and a special management for the missing signal intervals in the RT analysis task is necessary. However, the over-all usability of a diagnostic for the intended RT purpose is not compromised with rare frame-drops [11].

Besides frame-drops at the very beginning of DAQ, other time patterns of frame-drops (e.g. stochastic occurrences over the whole discharge) can be observed occasionally. This normally indicates a mistake in the priority set-up of the involved RT tasks or a mistake in the RT configuration parameters of the Solaris operating system. In cases where correcting such mistakes did not help, this was due either to using higher data rates than permitted by the constraints mentioned above or to employing a computer platform and/or configuration inappropriate to the problem. 
Figure 1 shows the appearance of the last described (stochastic) type of frame-drops over 700 AUG shot numbers during the lengthy (and partly cumbersome) tuning period of the RMC diagnostic. The reappearance of frame-drops on minor deviations from the optimal system parameter settings illustrates how sensitively a SIO I configuration operated near the performance limit (here RMC) depends on correct or ill-tuned RT settings. On the other hand, the results demonstrate the stability of SIO over longer time periods when system parameters are well tuned.

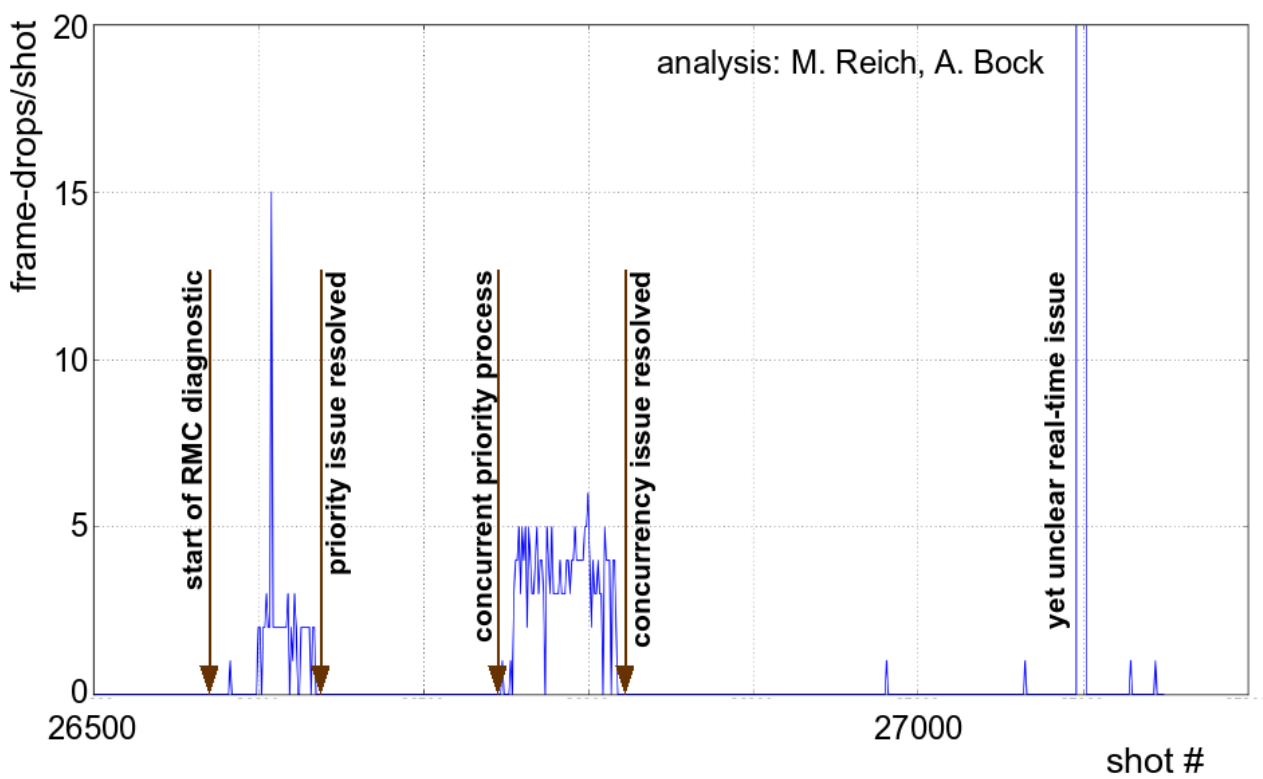

Figure 1: RMC diagnostic - SIO frame-drops per shot

\section{Investigating Solaris OS Interrupt Latency with dtrace}

When frame-drops first came into play it soon became clear how important the right choice of Operating System (OS) settings is for tuning the RT behavior for the processes and threads involved in SIO DAQ and RT analysis.

As illustrated above, the most crucial parameter here is the latency the OS incurs in handling DMA interrupts, that is, the delay time during which the DMA is not running between the end of the previous and the beginning of the next partial DMA read operation.

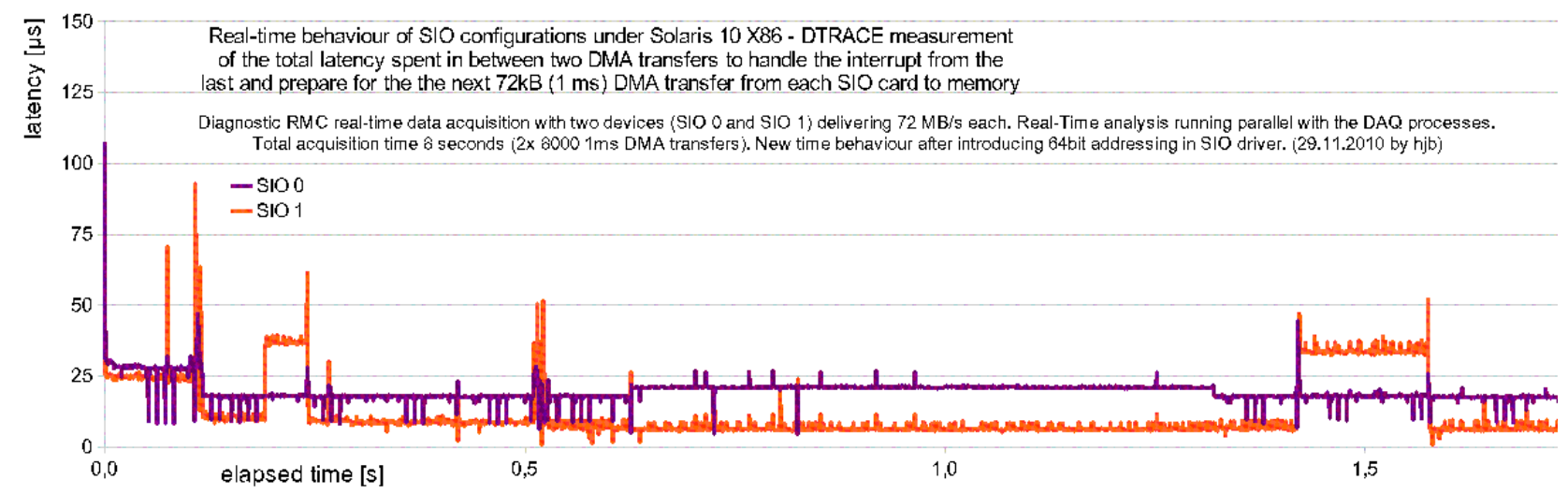

Figure 2: Solaris 10 real-time DMA interrupt handling latency measured for 8 seconds (1 irq/ms per device) for the RMC diagnostic with two SIO I cards (devices SIOO and SIO1) operating at a data rate of $72 \mathrm{MB} / \mathrm{s}$ each. (Here in fact the first $1.5 \mathrm{~s}$ are shown. The full high resolution figure as well as the D-script to do the dtrace measurement can be downloaded from the online annex material of the digital journal. [URL to be completed during the editing process of the paper, please find the high resolution figure as a separate PDF attached to the submission]) 
In summary: the achievable over-all DMA speed depends on the DMA duty cycle, whereas the occurrence of frame-drops depends on the reliable reproducibility of a latency which is short enough to avoid FIFO overflows.

Without going too deeply into the details, we may briefly discuss the results presented in Figure 2 from dtrace measurements [http://www.dtrace.org] of the behavior of Solaris OS under SIO operation. As can be seen from this figure, DMA latency for both SIO devices is typically less than $25 \mu$ s (which is in agreement with earlier results [1] sect. 3.6, fig. 7), time intervals where DMA latency extends up to $50 \mu$ s are short, one peak up to $150 \mu$ s near $2.9 \mathrm{~s}$ seems to be an isolated event. At the very beginning of the DMA chain a few long latency events are always observed. We suspecte these delays to be caused by some OS or processor activity (loading caches, preparing MMU tables) which are difficult to anticipate.

In comparison to these illustrated latency results, the buffering capability of the $32 \mathrm{kB}$ DMA FIFO in this case is enough to hold about $440 \mu$ s of data.

\section{SIO II prospects}

\section{Requirements and Hardware Redesign}

Up till here current and future diagnostic demands have been discussed in comparison to SIO I performance data. Diagnostic implementations, their deployment characteristics and difficulties have been presented. Performance demands beyond the current capabilities of SIO I have been identified.

Three main topics have to be considered to increase SIO performance:

1. More powerful FPGA

- SIO I is based on an Altera Cyclone II with a $50 \mathrm{MHz}$ clock which leads to an internal data transfer speed limit at approximately 120-140 MB/s.

- Using an Arria II GX instead would provide multiple clock domains with a base clock of $125 \mathrm{MHz}$. At a width of 8 bytes, the internal data transfer rate could reach a maximum of $1 \mathrm{~GB} / \mathrm{s}$.

2. Faster PCI interface to host

- SIO I with a cPCI or cPCIexpress x1 adapter is limited by the theoretical speed of its bus interface. If additionally a PCI/cPCI or a PCIe/cPCIe bridge is inserted between SIO and host, further limitations apply. Therefore the nominal host interface speed varies from 78 to $160 \mathrm{MB} / \mathrm{s}$. (N.b. further limits are imposed by the FPGA's internal limit.)

- Using the built-in PCIexpress-Hard-IP of the Arria II GX enables state of the art, high-speed, multi-lane PCIe data rates up to several Gb/s. Indeed, the FPGA's PCIexpress interface is expected no longer to create significant bottlenecks of its own.

3. Faster serial links

- SIO I provides four HOTlink II connections to the DAQ front-ends, each being able to transfer up to $40 \mathrm{MB} / \mathrm{s}$. The HOTlink II protocol for these links is provided by a separate chip requiring additional considerations. Not only the demand for more speed but also the possible integration of the serial links as well as an internal FPGA function block justify a more far-reaching design change. The advantage of simplifying the hardware design compensates for violating compatibility with the existing front-end controllers. In consequence, a new design of this front-end 
counterpart of the SIO card is probably unavoidable, but the modular design of most front-ends requires exchanging one card per crate only.

- The above mentioned FPGA series provide their own serial link concept, IP SerialLite II [5]. The nominal speed of these links is specified as $2.5 \mathrm{~Gb} / \mathrm{s}$ which is approximately six times faster than HOTlink II. Therefore, we have decided to change the serial protocol between future SIO cards and DAQ front-ends to SerialLite II.

The redesign of the front-end controller card with an Altera FPGA to implement the "pipe line" front-end (c.f. [1]) control logic was expected to be straightforward and has successfully been completed.

Further requirements have been collected:

- functional compatibility to SIO I

$\circ$ with the modular PIPE front-end system

- with the ASDEX Upgrade timing system

- extendability of the functions implemented in the FPGA

- implement further DAQ modes

- RT processing of data streams

- long-term market support of the chosen interfaces

- universal form factor, compact board size

- FPGA firmware in place programmable

- affordable price

Taking all the above considerations into account, an SIO II prototype was built as an XMC form factor mezzanine board. Figure 3 depicts the schematic hardware layout. The FPGA is flanked by

XMC-Modul

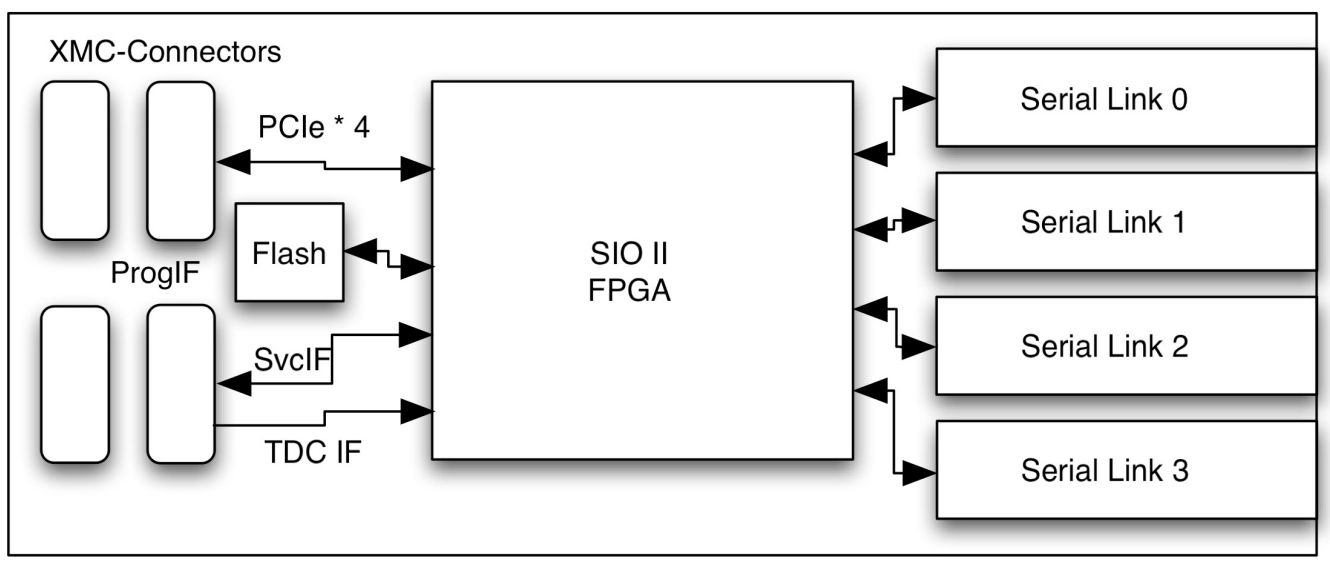

Figure 3: SIO II XMC board layout diagram

the optical transmitters on the right side and the XMC connectors to the carrier board and further to the host on the left. While the FPGA flash memory is shown, all other supporting components have been omitted from this figure. 


\section{FPGA Software Redesign}

The described redesign decisions for SIO II have also made necessary a revision of the internal FPGA software layout. An "SOPC" (System On Programmable Chip) approach has been chosen. Keeping all components within one chip avoids difficult high frequency PCB design issues. Together with a very flexible reprogrammability, the system facilitates an outstanding adaptability to future needs. The software layout features a central communication fabric and various function blocks arranged accordingly as Figure 4 depicts. The upper part displays the TDC timing module, four serial link modules, and in between the transfer module derived from the so called "merge engine" of SIO I. Together these three implement the core SIO engine. Below the central "Interconnect Fabric" the host side modules are shown. Beside the PCIe controller block, the DMA engine, addressing table memory, and FPGA control \& status memory and registers are shown. The

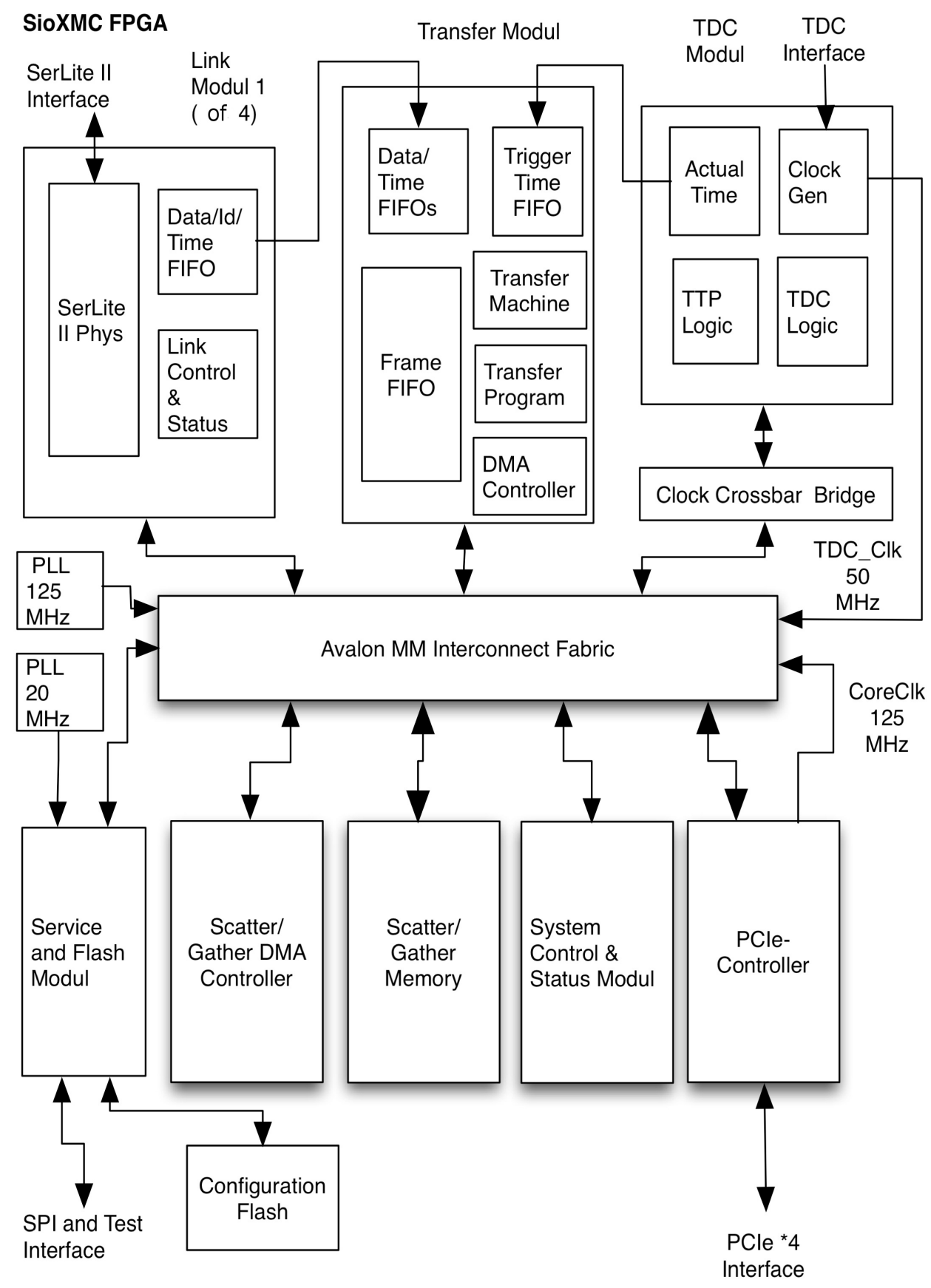

Figure 4: SIO II FPGA "system on a programmable chip" layout diagram 
"Service and Flash Module" implements the possibility to update the FPGA program in situ via the host interface.

\section{First Prototype of SIO II}

Together with the SIO II XMC mezzanine board a PCIe carrier board was prototyped. Figure 5 shows both boards. On top on the left the component side view (mirrored to the schematic in Figure 4) shows the four optical transmitters for the serial links. These occupy the entire available XMC

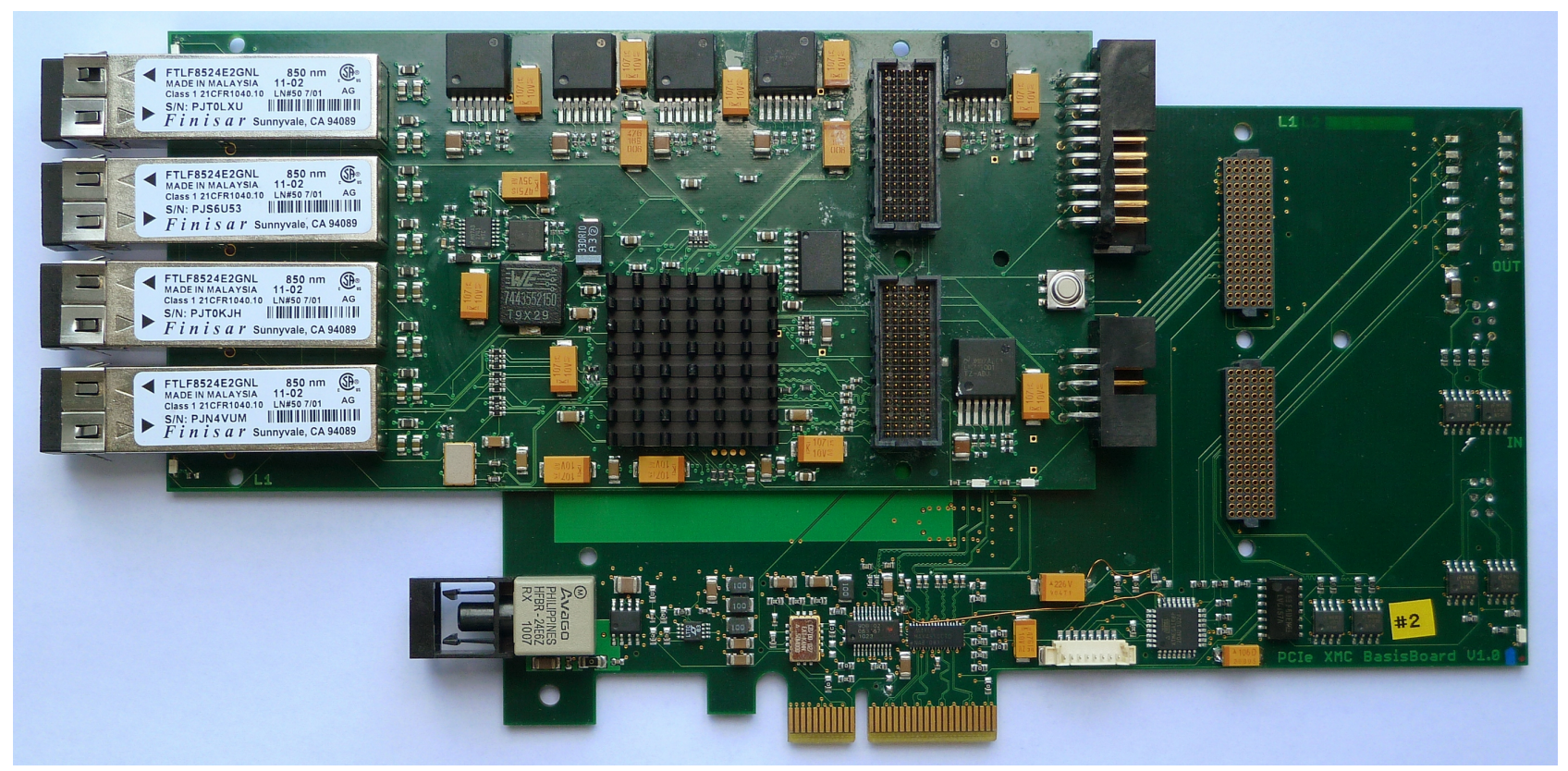

Figure 5: SIO II XMC prototype (foreground) and XMC/PCIe carrier board (background)

front-panel space. The FPGA in the middle is covered by a heat sink. Beside and above the FPGA the already mentioned flash memory and a couple of DC/DC power supply chips can be seen. To the right of the FPGA the XMC connectors providing high frequency links to the carrier board are located. On the very right edge of the mezzanine, diagnose and programming connectors are visible.

The ASDEX Upgrade timing network receiver is not part of the SIO II card itself. Instead it is located on the PCIe carrier board, which is shown in the same figure behind the SIO II board. The carrier board merely consists of a transparent PCIexpress bridge and the optical AUG time receiver. The receiver converts the fiber optics time signal to the Manchester-Coded AUG experiment clock[12]. This clock is provided to the SIO FPGA by the carrier board via the XMC connector. Conveniently, multiple SIO II XMC's may share the same external clock receiver. Furthermore, other timing networks could be used if the respective carrier board provides the timing clock via the XMC carrier connector and the FPGA's timing software is adapted to handle the corresponding time representation.

As an XMC mezzanine, SIO II is designed to be useable on various other carrier platforms if desired. Most likely these could be compact PCIexpress, PXI, or ATCA carrier boards aggregating one or more SIO II in one host slot. To adapt to future, faster PCIe versions an FPGA software upgrade or a slight redesign is expected to be sufficient.

The illustrated PCIe carrier bridge features four PCIexpress lanes, thus permitting up to $1 \mathrm{~GB} / \mathrm{s}$ DMA data rates from SIO II to host memory. In first trials, the SIO II prototype achieved a data rate of $480 \mathrm{MB} / \mathrm{s}$ from FPGA to host memory. 


\section{Conclusions}

Serial I/O low latency delivery of data direct into the memory of computers as DAQ concept emerged a couple of years ago from previous ideas (see [1]). Since then, SIO has become a new diagnostics standard at ASDEX Upgrade. Deployed successfully in a series of diagnostics, the SIO approach demonstrates power and versatility for real-time and classical DAQ applications. This success together with demanding requirements in prospect of future diagnostic needs has led to a definition and new design of SIO II as a next step serial I/O interface card. A first prototype is currently being tested with promising results. Among these, a measured throughput of $480 \mathrm{MB} / \mathrm{s}$ seems to be most noteworthy.

We are planning to upgrade and condense a series of diagnostics which are either still distributed among a bunch of computer nodes (in the long-run these will be the Soft-X-Ray and Mirnov Probe diagnostics; in the short term, several CAMAC-based diagnostics will be refurbished using the SIO solution), or have not yet been capable of handling the sampling rates we were aspiring to for years (e.g. an upgrade of ECE from 1 to $2 \mathrm{MHz}$ as well as an upgrade of at least a part of the Langmuir probe channels to $2-4 \mathrm{MHz}$ sampling rate).

\section{Acknowledgment}

We want to thank our colleagues from the ASDEX Upgrade team for their encouraging contributions in testing and tuning the new diagnostics. Especially M. Reich and G. Conway had a lot extra work to get things up and running as well as adapting their preparation and analysis programs carefully to the new modus operandi SIO is bringing in.

We also would like to thank Constantin Gonzales from Oracle (former SUN Microsystems) who spent a day with us breeding over Solaris performance issues and how to unleash the power of dtrace for our investigations.

\section{References}

[1] K. Behler, H. Blank, H. Eixenberger, A. Lohs, K. Lüddecke, R. Merkel, G. Raupp, G. Schramm, W. Treutterer, M. Zilker, ASDEX Upgrade Team, Real-Time Diagnostics at ASDEX Upgrade - Architecture and Operation, Fusion Engineering and Design 83 (2008) 304-311, http://dx.doi.org/10.1016/j.fusengdes.2008.01.015

[2] K. Behler, H. Blank, A. Buhler, R. Cole, R. Drube, K. Engelhardt, H. Eixenberger, N.K. Hicks, A. Lohs, K. Lüddecke, A. Mlynek, U. Mszanowski, R. Merkel, G. Neu, G. Raupp, M. Reich, W. Suttrop, W. Treutterer, M. Zilker, ASDEX Upgrade Team, Real-Time diagnostics at ASDEX Upgrade - Architecture and operation, Fusion Engineering and Design 85 (2010) 313-320, http://dx.doi.org/10.1016/j.fusengdes.2010.03.001

[3] Daniel Charlet, P. Abbon, R. Ansari, C. Beigbeder, D. Breton, T. Cacérès, H. Deschamps, C. Flouzat,P. Kestener, C. Magneville, B. Mansoux, C. Pailler, M. Taurigna, and C. Yèche, The BAO Radio Acquisition System, IEEE Transactions on Nuclear Science 58 (2011) 1833-1837, http://dx.doi.org/10.1109/TNS.2011.2155085

[4] Altera Corporation, Arria II FPGAs: Cost-Optimized, Lowest Power 6G Transceiver

FPGAs, http://www.altera.com/devices/fpga/arria-fpgas/arria-ii-gx/aiigx-index.jsp (visited 2012-08-

[5] Altera Corporation, Altera's Complete SerialLite II Solution, http://www.altera.com/technology/high_speed/protocols/seriallite/pro-seriallite.html (visited 201208-22)

[6] Altera Corporation, Altera's Intellectual Properties PCI Compiler, http://www.altera.com/products/ip/iup/pci/m-alt-pci_mt32.html (visited 2012-08-28) 
[7] A. Mlynek, M. Reich, L. Giannone, W. Treutterer, K. Behler, H. Blank, A. Buhler, R. Cole, H. Eixenberger, R. Fischer, A. Lohs, K. Lüddecke, R. Merkel, G. Neu, F. Ryter, D. Zasche, ASDEX Upgrade Team, Real-time feedback control of the plasma density profile on ASDEX Upgrade, Nuclear Fusion 51 (2011) 043002, http://dx.doi.org/10.1088/0029-5515/51/4/043002

[8] N.K. Hicks, W. Suttrop, K. Behler, M. García-Muñoz, L. Giannone, M. Maraschek, G. Raupp, M. Reich, A.C.C. Sips, J. Stober, ASDEX Upgrade Team, S. Cirant, G. D'Antona, Fast Sampling Upgrade ans Real-Time NTM Control Application of the ECE Radiometer on ASDEX Upgrade, Fusion Science and Technology 57 (2010) 1-9, http://www.new.ans.org/pubs/journals/fst/a_9263

[9] M. Reich, K. Behler, R. Drube, L. Giannone, A. Kallenbach, A. Mlynek, J. Stober, W. Treutterer, ASDEX Upgrade Team, Real-Time diagnostics and their Applications at ASDEX Upgrade, Fusion Science and Technology 58 (2010) 727-732,

http://www.new.ans.org/pubs/journals/fst/a_10921

[10] Wolfgang Treutterer, Gregor Neu, Gerhard Raupp, Thomas Zehetbauer,Dieter Zasche, Klaus Lüddecke, Richard Cole, ASDEX Upgrade Team, Real-time signal communication between diagnostic and control in ASDEX Upgrade, Fusion Engineering and Design 85 (2010) 466-469, http://dx.doi.org/10.1016/j.fusengdes.2010.04.031

[11] M. Reich, A. Bock, M. Maraschek, ASDEX Upgrade Team, NTM Localization by Correlation of Te and dB/dt, Fusion Science and Technology 61 (2012) 309-313,

[12] G. Raupp, R. Cole, K. Behler, M. Fitzek, P. Heimann, A. Lohs, K. Lüddecke, G. Neu, J. Schacht, W. Treutterer, D. Zasche, Th. Zehetbauer, M. Zilker, A "Universal Time" system for ASDEX Upgrade, Fusion Engineering and Design 66-68 (2003) 947-951,

\section{Table Captions}

Table 1: Recently deployed SIO I diagnostics at ASDEX Upgrade.

\section{Figure Captions}

Figure 1: RMC diagnostic - SIO frame-drops per shot.

Figure 2: Solaris 10 real-time DMA interrupt handling latency measured for 8 seconds $(1 \mathrm{irq} / \mathrm{ms}$ per device) for the RMC diagnostic with two SIO cards operating at a data rate of $72 \mathrm{MB} / \mathrm{s}$ each. (Here in fact the first $1.5 \mathrm{~s}$ are shown. The full high resolution figure as well as the D-script to do the dtrace measurement can be downloaded from the online annex material of the digital journal. [URL to be completed during the editing process of the paper, please find the high resolution figure as a separate PDF attached to the submission]).

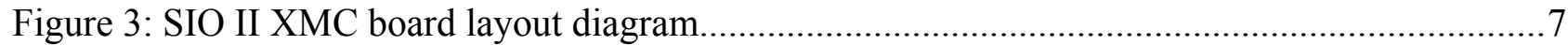

Figure 4: SIO II FPGA "system on a programmable chip" layout diagram..................................8

Figure 5: SIO II XMC prototype (foreground) and XMC/PCIe carrier board (background).............9 


\section{Annex material to be provided on-line}

1. Full resolution version of Figure 2 provided with the submission as separate PDF-file.

2. dtrace script to do the DMA latency measurements

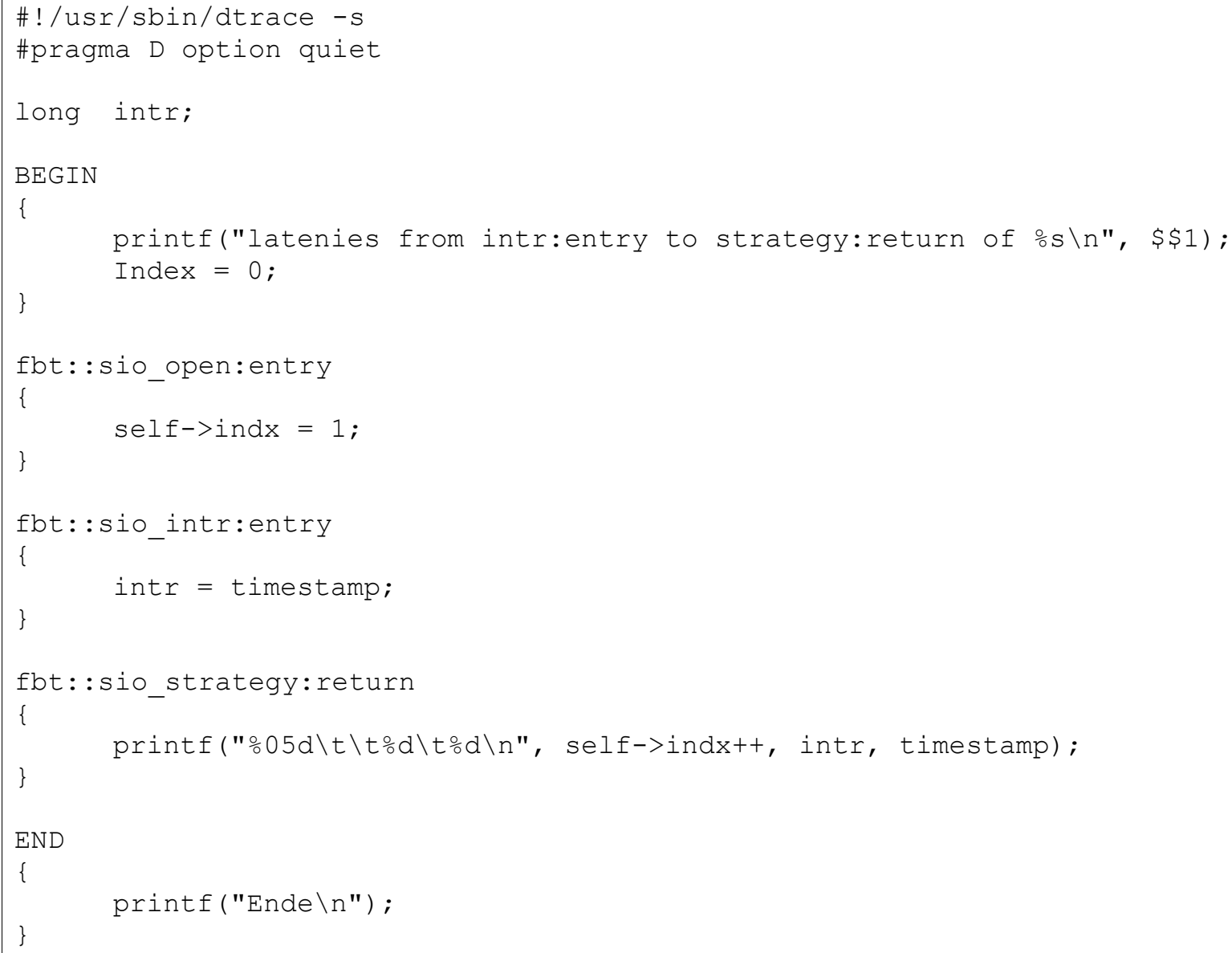

3. All figures and tables to be printed in the journal in b/w or gray scale should be provided online in color. Please refer to the following pages containing large scale versions of the figures. 


\section{Tables}

\begin{tabular}{|c|c|c|c|c|c|c|c|}
\hline Diagnostic & CPU cores bus & \# SIO/links & pipelines $x$ slots & DAQ modules & sample rate & data rate & data size \\
\hline$r$-t $n_{e}$ Interferom. (DCN) & 1x sparcV9 $\mathrm{PCl}$ & $1 / 1$ & $1 \times 12$ & $5 x F r g C n t+3 x 2$ chADCs & $50 \mathrm{kHz}$ & $1.6 \mathrm{MB} / \mathrm{s}$ & 17 \\
\hline r-t current profile (MSE) & d64 PCle & $1 / 1$ & $1 \times 16$ & 13x2chADCs & $250 \mathrm{kHz}$ & $15 \mathrm{MB} / \mathrm{s}$ & 180 \\
\hline ECE (RMC) & 2x4 amd64 PCle & $2 / 4+4$ & $2 \times 4 \times 2$ & $8 \times 2 \times 4 c h A D C$ (wls) & $1 \mathrm{MHz}$ & $2 \times 72 \mathrm{MB} / \mathrm{s}$ & \\
\hline XUV emission (XVR) & 2x sparcV9 PCle & $2 / 4+4$ & $2 \times 4 \times 16$ & 128x XUV-ADCs & $500 \mathrm{kHz}$ & $2 \times 68 \mathrm{MB} / \mathrm{s}$ & $1 \mathrm{G}$ \\
\hline XUV emission (XVS) & 1x sparcV9 PCle & $2 / 4+3$ & $(4+3) \times 16$ & 112x XUV-ADCs & $500 \mathrm{kHz}$ & $68+52 \mathrm{MB} / \mathrm{s}$ & 960 \\
\hline VUV Spectr. (CCD) & $\mathrm{cV} 9 \mathrm{PCl}$ & $4 / 1$ & custom CCD FE & 4x CCD-HL Adapter & $1 \mathrm{kHz}$ & $6 \mathrm{MB} / \mathrm{s}$ & 24 \\
\hline Fast Ion Loss & 1x sparcV9 $\mathrm{PCl}$ & $1 / 3$ & $3 \times 4$ & $9 x$ 2chADCs & $1 \mathrm{MHz}$ & $44 \mathrm{MB} / \mathrm{s}$ & 352 \\
\hline Lithium Beam & 2x4 amd64 PCle & $1 / 3$ & $3 \times 16$ & $48 \times 2$ chADCs & $200 \mathrm{kHz}$ & $40 \mathrm{MB} / \mathrm{s}$ & $27 \mathrm{M}$ \\
\hline Reflectometry fl & 2x4 amd64 PCle & $1 / 1$ & $1 \times 4$ & $2 \times 2$ chADCs & $1 \mathrm{MHz}$ & $16 \mathrm{MB} / \mathrm{s}$ & $128 \mathrm{M}$ \\
\hline Doppler Reflectometry & 2x4 amd64 PCle & $2 / 2$ & custom FE & 2 bands ( $2 x 2$ ADCs) & $20 \mathrm{MHz}$ & $2 \times 80 \mathrm{MB} / \mathrm{s}$ & 1.2 \\
\hline
\end{tabular}


Figures

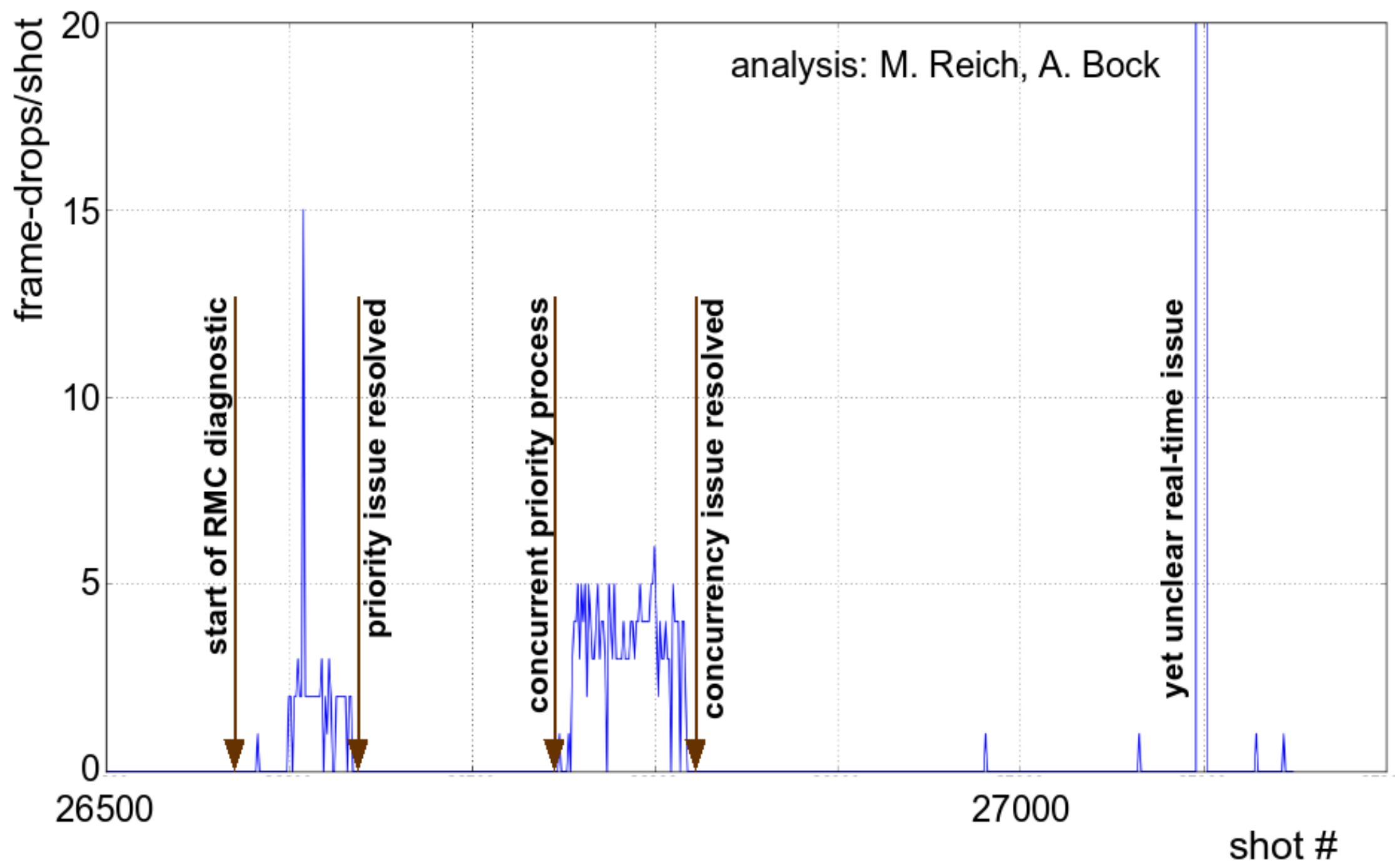

Figure 1: RMC diagnostic - SIO frame-drops per shot 


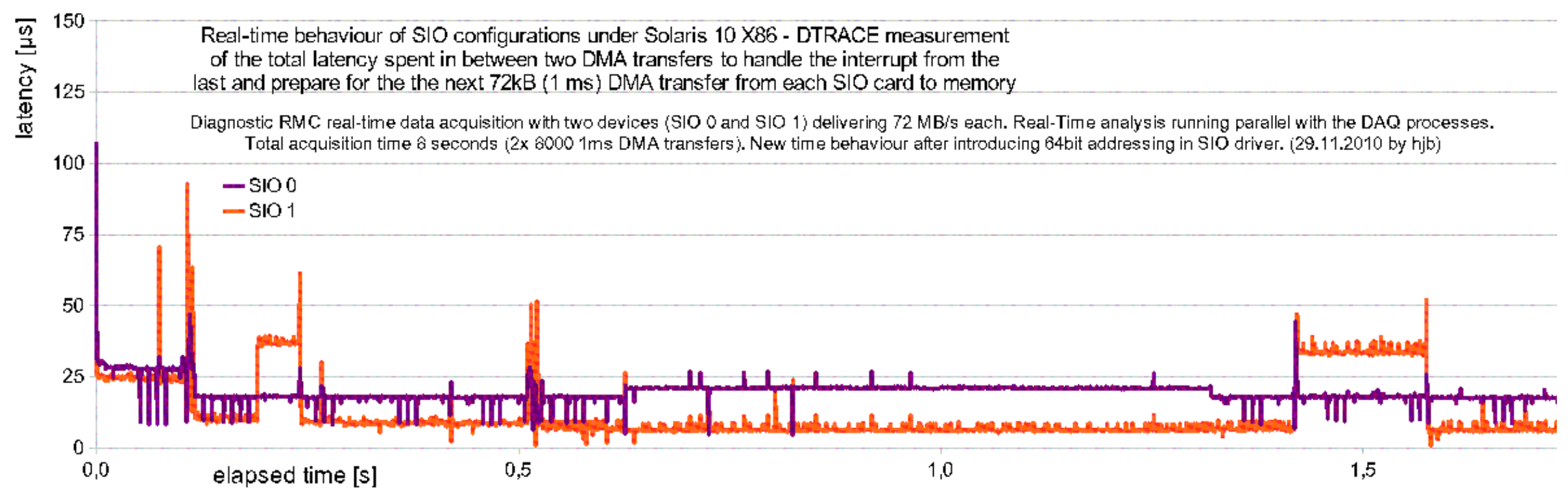

Figure 2: Solaris 10 real-time DMA interrupt handling latency measured for 8 seconds (1 irq/ms per device) for the RMC diagnostic with two SIO cards operating at a data rate of $72 \mathrm{MB} / \mathrm{s}$ each. (Here in fact the first $1.5 \mathrm{~s}$ are shown. The full high resolution figure as well as the D-script to do the dtrace measurement can be downloaded from the online annex material of the digital journal. [URL to be completed during the editing process of the paper, please find the high resolution figure as a separate PDF attached to the submission]) 


\section{XMC-Modul}

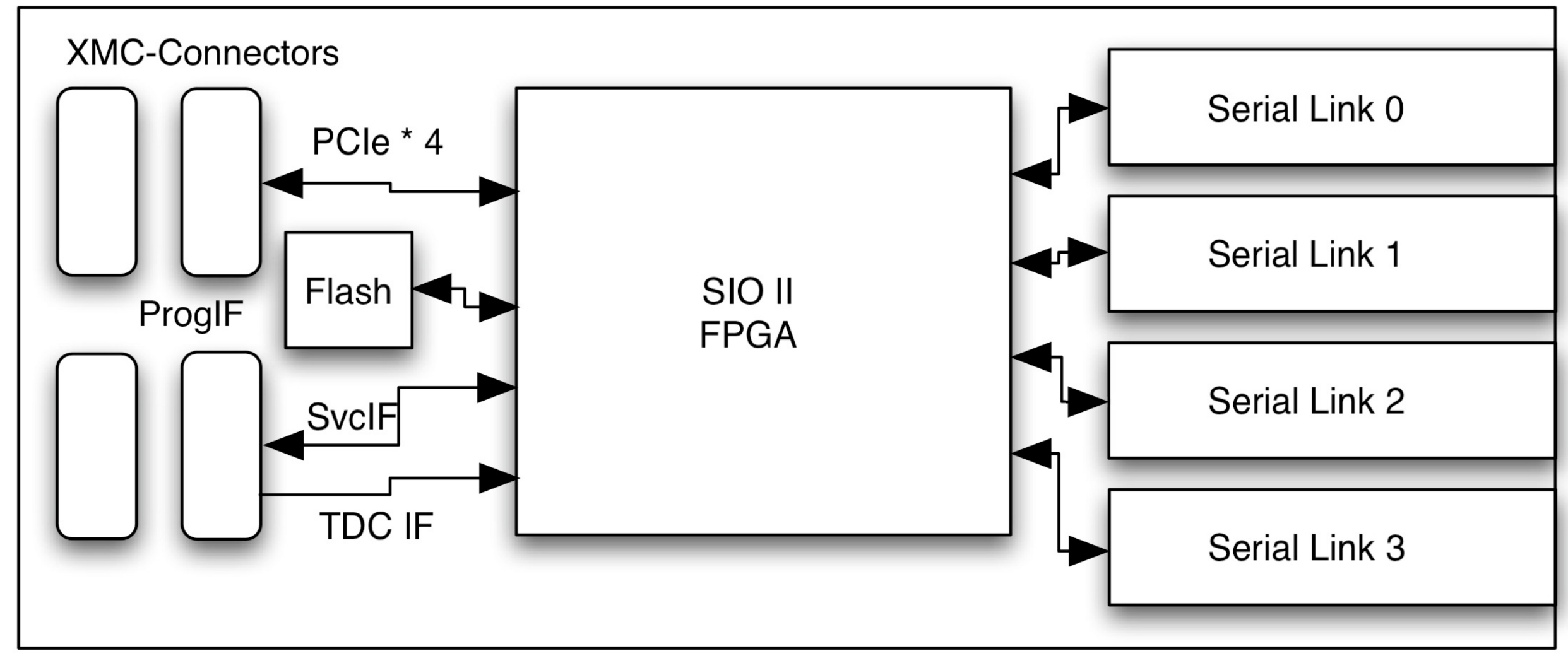

Figure 3: SIO II XMC board layout diagram 


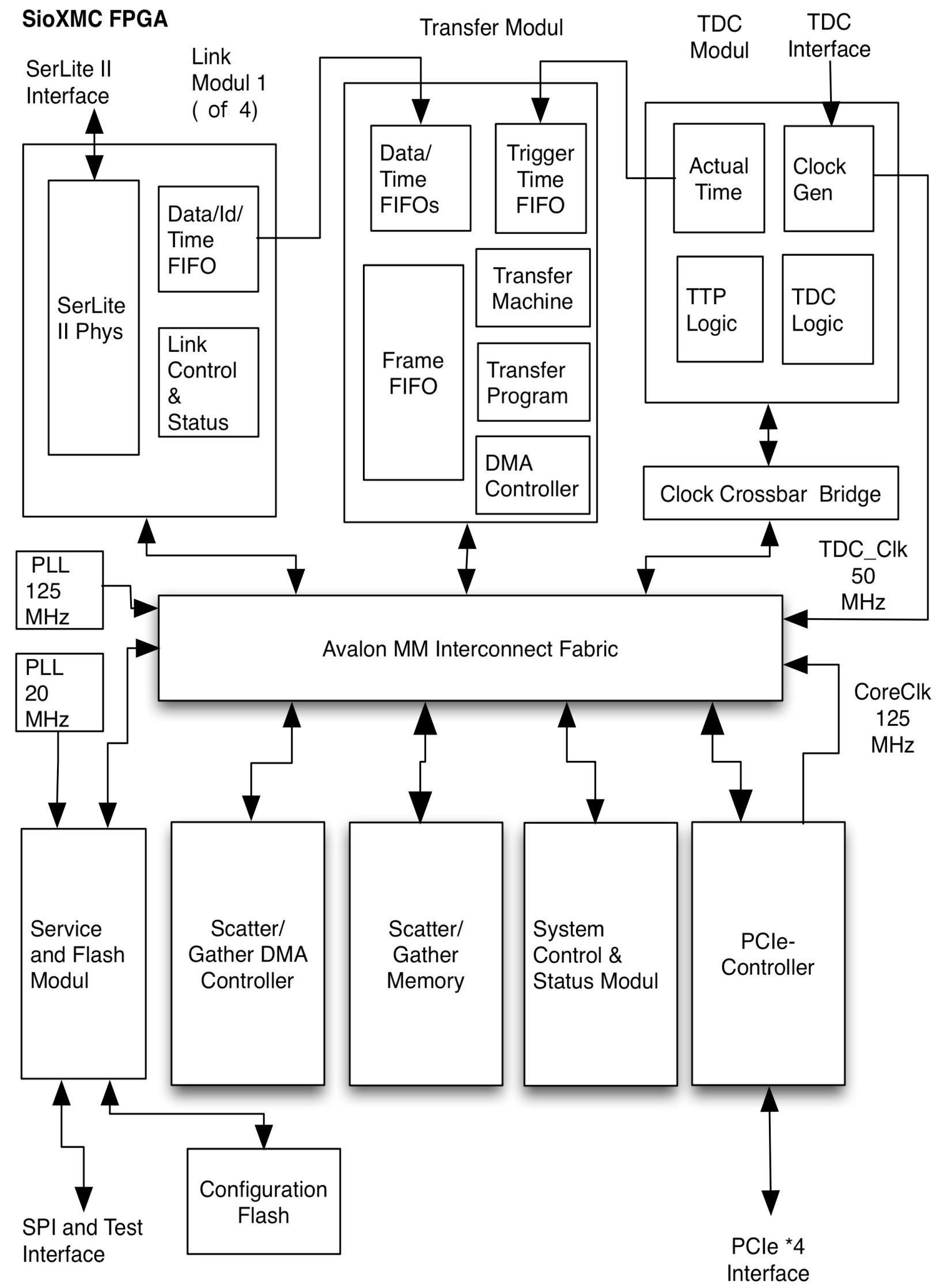

Figure 4: SIO II FPGA "system on a programmable chip" layout diagram 


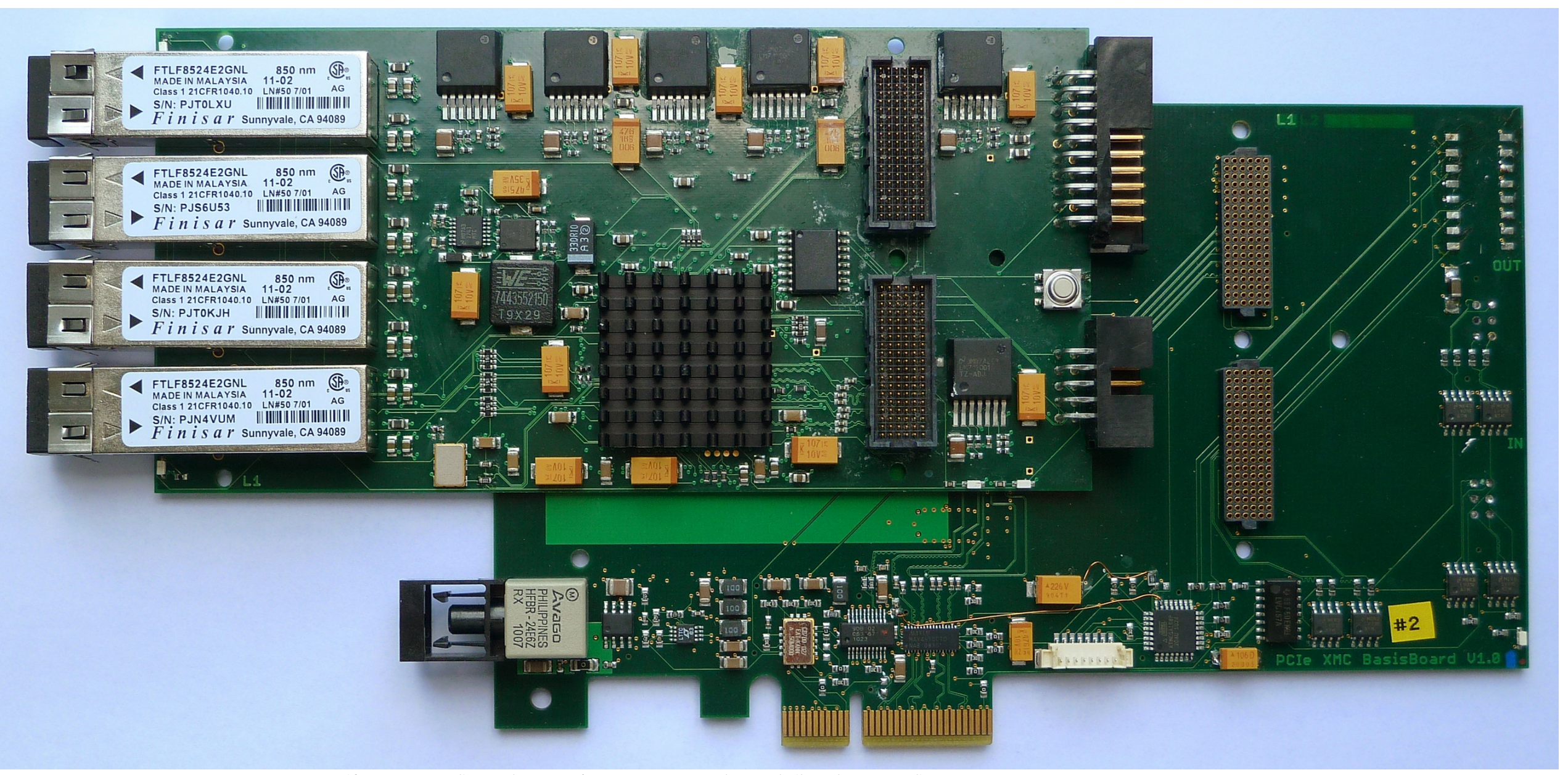

Figure 5: SIO II XMC prototype (foreground) and XMC/PCIe carrier board (background) 
